Voix et Images

\title{
Analyse de textes - Réjean Ducharme et Victor-Lévy Beaulieu : les mots et les choses
}

\section{André Vanasse}

Volume 3, numéro 2, décembre 1977

Victor-Lévy Beaulieu

URI : https://id.erudit.org/iderudit/200104ar

DOI : https://doi.org/10.7202/200104ar

Aller au sommaire du numéro

Éditeur(s)

Les Presses de l'Université du Québec

ISSN

0318-9201 (imprimé)

1705-933X (numérique)

Découvrir la revue

\section{Citer cet article}

Vanasse, A. (1977). Analyse de textes — Réjean Ducharme et Victor-Lévy

Beaulieu : les mots et les choses. Voix et Images, 3(2), 230-243.

https://doi.org/10.7202/200104ar d'utilisation que vous pouvez consulter en ligne.

https://apropos.erudit.org/fr/usagers/politique-dutilisation/ 


\section{Analyse de textes \\ Réjean Ducharme et Victor-Lévy Beaulieu : les mots et les choses}

\section{Préambule}

A peu près à la même époque où Ferdinand de Saussure élaborait les concepts de signifiant et signifié dans la description du signe linguistique, Freud mettait de l'avant les concepts de «représentation de mot» et "représentation de chose" par lesquels il voulait signifier que les effets de sens pouvaient être pris en charge soit par une certaine représentation psychique du mot (le son, la lettre...) soit par la représentation de la chose. C'est d'ailleurs à partir de cette double description que Freud avait pu différencier le schizophrène du paranoïaque où il démontrait que le premier surinvestissait les représentations de mots tandis que le second s'accrochait plutôt aux représentations de choses. Ces deux concepts se distinguent à peine de ceux énoncés par Saussure; ils pourraient éventuellement être d'une certaine utilité pour l'étude des textes littéraires.

J'essaierai donc dans ce court article de définir deux types d'écriture (écriture schizoïde et écriture paranoïde) en faisant appel à la théorie psychanalytique. Pour éviter toute ambiguïté qu'il soit entendu que l'emploi des termes "mots" et "choses" signifient "représentations de mot ou de chose" puisqu'il est clair pour moi, comme pour les linguistes, que "le signe linguistique [c'est celui que j'étudie dans cet article] unit non une chose et un nom mais un concept et une image acoustique ${ }^{1}$ '.

\section{Les mots contre les choses}

Le choix de Réjean Ducharme et de Victor-Lévy Beaulieu comme terrain d'analyse pour illustrer ma typologie est loin d'être innocent. En tant que lecteur, je situe ces deux auteurs aux antipodes. Cette affirmation repose sur une perception intuitive mais qui est tout de même fondée sur un certain nombre d'indices probants.

Ainsi les articles écrits sur Réjean Ducharme tentent, pour la plupart, de mettre en valeur le travail constant du signifiant. L'article de Gilles Marcotte, "Réjean Ducharme contre Blasey Blasey ${ }^{2}$ " et celui de Bernard Dupriez “Ducharme et des ficelles ${ }^{3}$ ” sont éloquents à ce sujet. Non seulement insistent-ils sur le travail du signifiant mais ils sont eux-mêmes pri- 
sonniers des pièges tendus par Ducharme, à ce point que leurs titres d'article sont mimétiques: Marcotte oppose Ducharme aux "blasés " tandis que Dupriez, un peu plus discret dans ses anagrammes nous donne à lire que «Ducharme est difficile».

Par ailleurs les articles qui traitent de la production de Victor-Lévy Beaulieu portent en général sur le signifié «caché» de l'œuvre. A preuve les analyses psycho-critiques de Bessette ${ }^{4}$ et moi-même ${ }^{5}$ ou encore les commentaires de Gabrielle Poulin 6 : dans tous les cas il s'agit d'analyses de contenu où plus souvent qu'autrement le signifiant est laissé pour compte.

A partir des remarques qui précèdent, on est en droit de penser que l'écriture d'un romancier puisse favoriser une face ou l'autre du signe linguistique. Personnellement j'ai toujours cru que, par exemple, Hubert Aquin était dans la même lignée que Ducharme et que, à l'opposé, Anne Hébert se rangeait du côté de Victor-Lévy Beaulieu. Ces hypothèses devront être vérifiées et, éventuellement, rectifiées mais on ne peut nier catégoriquement qu'il y ait une certaine vérité dans cette affirmation.

Sans doute pourrait-on allonger la liste et constater que d'autres auteurs ne coïncident pas avec ces deux extrêmes que sont Ducharme et Beaulieu. Gérard Bessette et Gilbert Larocque, pour ne citer qu'eux, appartiennent à une autre catégorie où signifiant et signifié sont moins visiblement dissociés. J'espère un jour revenir là-dessus.

Pour l'instant il est permis d'affirmer sans risque d'erreur grave que l'écriture de Ducharme repose principalement sur les effets de sens produits par les mots. Les titres de ses romans, l'Avalée des avalés, l'Océantume, Le nez qui voque, pour ne nommer que les plus manifestes, ne laissent aucun doute à ce sujet: Ducharme a pour parti pris esthétique de rendre les mots opaques, de faire du mot un obstacle. On a, bien avant moi, montré comment le texte de Ducharme se veut polysémique. Lire Ducharme c'est d'abord jouer du dictionnaire et de l'encyclopédie. Les mots sont déformés (les dates aussi) à commencer par les titres: Comment lire «l'Océantume»? S'agit-il de “l'Amertume»? de «La mer (ou l'océan) t'hume»? De «Le séant t'hume»? etc., etc. On pourrait par centaines, par milliers même, multiplier les exemples pour la bonne raison que le texte de Ducharme fonctionne d'abord et avant tout sur la mise en valeur des innombrables possibilités du signifiant.

Chez Victor-Lévy Beaulieu le texte s'organise plutôt à partir de la transparence du mot. Cela ne veut pas dire que Beaulieu ne cherche pas à créer son propre style, bien au contraire, mais que sa pratique de l'écriture se modèle sur celle d'un désir, avoué ou pas, de conformité avec les choses. Si l'on accepte quelques tics littéraires artificiels et agaçants (une cerțaine obstination à déformer la graphie de certains mots: par exemple «fesant" pour "faisant", ou «ouique» pour "week») Victor-Lévy Beaulieu apparaît naturellement comme le pratiquant d'une écriture «blanche». 
II est évident, pour quiconque a lu avec un minimum d'attention cet auteur, qu'il privilégie le parti pris des choses. Beaulieu est dans une certaine mesure l'écrivain de la scatologie: la merde, la vomissure, la pisse, le sperme barbouillent son texte à ce point que plusieurs le trouvent inacceptable! Ducharme de son côté circule dans le monde clos des mots. Chez lui la vulgarité est bannie. On peut certes à l'occasion l'accuser de mauvais goût (ses jeux de mots sont parfois puérils pour ne pas dire insignifiants) mais dans l'ensemble le texte de Ducharme évite les grossièretés que se permet si allègrement Beaulieu.

La meilleure façon d'illustrer les différences de "style » entre Ducharme et Beaulieu consiste à les soumettre à une analyse. J'ai choisi mes textes au hasard (un hasard qui peut être sujet à caution!): il s'agit des débuts de l'Avalée des avalés et de Don Quichotte de la démanche.

Voici donc, en parallèle, les deux textes:

\section{V.-L. Beaulieu}

Et puis il comprit qu'il allait mourir au beau milieu d'une phrase, alors qu'il cherchait ses mots et n'était pas satisfait de ceux qu'il trouvait: ils étaient ou bien trop longs (serpents à lettres pâteuses qui entouraient ses jambes et faisaient monter le sang à sa tête) ou bien creux comme le tronc du vieil érable qu'avec son père jurant et crachant le brun-malade de sa chique, il avait abattu jadis devant la maison lambrissée de papierbrique, à Saint-Jean-de-Dieu. Ou bien encore les mots brillaient trop devant ses yeux, l'éblouissaient, lui donnaient le vertige, se modifiaient en d'apeurantes étoiles noires qui glissaient silencieusement derrière ses paupières closes. Alors Abel se laissait tomber et se mettait à hoqueter: désormais il n'allait plus pouvoir rien faire; quelque chose en lui (mais peut-être aussi cela venait-il de l'extérieur, dans cette fourmi absurde se frappant obstinément la tête contre la vitre de la fenêtre dans une entreprise désespérée tout autant qu'insensée, et dehors les sous-vêtements bruns battaient au vent, faisant quelque danse obscène sur la corde à

\section{R. Ducharme}

Tout m'avale. Quand j'ai les yeux fermés c'est par mon ventre que je suis avalée, c'est dans mon ventre que j'étouffe. Quand j'ai les yeux ouverts, c'est parce que je vois que je suis avalée, c'est dans le ventre de ce que je vois que je suffoque. Je suis avalée par le fleuve trop grand, par le ciel trop haut par les fleurs trop fragiles par les papillons trop craintifs, par le visage trop beau de ma mère. Le visage de ma mère est beau pour rien. S'il etait laid, il serait laid pour rien. Les visages beaux ou laids, ne servent à rien. On regarde un visage, un papillon, une fleur et ça nous travaille, puis ça nous irrite. $\mathrm{Si}^{\text {y }}$ on $\mathrm{se}$ laisse faire, ça nous désespère. II ne devrait pas y avoir de visages, de papillons, de fleurs. Que j'aie les yeux ouverts ou fermés, je suis englobée: il n'y a plus assez d'air tout à coup, mon coeur se serre, la peur me saisit.

L'été les arbres sont habillés. L'hiver les arbres sont nus comme des vers. Ils disent que les morts mangent les pissenlits par la racine. Le jardinier a trouvé deux vieux tonneaux dans son grenier. Savez-vous ce qu'il en a fait? II les a sciés en deux pour en faire quatre seaux. II en a mis un sur la plage et trois dans le champ. Quand ils ont soif, les oiseaux s'arrêtent de voler et viennent y boire.

Je suis seule et j'ai peur. Quand j'ai faim, je mange des pissenlits par la racine et ça se passe. Quand j'ai soif, je plonge mon visage dans l'un des seaux et j'aspire. Mes cheveux déboulent dans l'eau. J'aspire et 
linge, provoquant l'œil, créant dans la cour un champ d'angoisse parfaitement circulaire et au centre duquel, comme quelque bête suant et jurant, lui, Abel Beauchemin venait de comprendre que jamais plus il ne pourrait écrire de romans).

Don Quichotte de la démanche, Montréal, L'Aurore, 1974, p. 13. ça se passe: je n'ai plus soif, c'est comme si je n'avais jamais eu soif. On aimerait avoir aussi soif qu'il y a d'eau dans le fleuve. Mais on boit un verre d'eau et on n'a plus soif. L'hiver, quand j'ai froid, je rentre et je mets mon gros chandail bleu. Je ressors, je recommence à jouer dans la neige, et je n'ai plus froid. L'été, quand j'ai chaud, j'enlève ma robe. Ma robe ne me colle plus à la peau et je suis bien, et je me mets à courir... (On ne peut rien contre la solitude et la peur. Rien ne peut aider. La faim et la soif ont leurs pissenlits et leurs eaux de pluie. La solitude et la peur n'ont rien. Plus on essaie de les calmer, plus elles se démènent, plus elles crient, plus elles brûlent. L'azur s'écroule, les continents s'abîment: on reste dans le vide, seul).

L'Avalée des avalés, Paris, Gallimard, 1966, p. 7-8.

Qu'y a-t-il donc de commun entre ces deux textes outre le fait qu'ils soient l'entrée en matière d'un roman? De fait, tous deux décrivent une situation extrême. Dans un cas, le personnage principal (Abel) constate subitement qu'il va mourir, dans l'autre la narratrice (nous apprendrons plus tard qu'il s'agit de Bérénice) décrit une situation presque aussi tragique qui est celle de l'avalement ( " tout m'avale ", «je suis englobée ", «j'étouffe», «je suffoque»...).

Par ailleurs, dans une perspective plus large, il est évident que les deux narrateurs décrivent leur relation au monde c'est-à-dire un certain système de représentation du réel.

Ce sont là, me semble-t-il, les trois points communs du texte. Les différences, elles, relèvent de l'écriture. Si les deux auteurs nous donnent à lire un certain réel, leur façon de le percevoir, ou plutôt le fait de le traduire par l'écriture, diffère totalement. Pourtant dans les deux cas il s'agit d'une description de choses concrètes. Au «tronc», à la «fourmi », à la "chaise" ou aux "sous-vêtements" du texte de Beaulieu, s'opposent le «fleuve», le «ciel», les «papillons» ou «le visage de ma mère» de celui de Ducharme.

\section{Ducharme: le parti pris des mots}

Quand on y regarde de près par contre on constate bien que la relation au monde de l'un est tout à fait opposée à celle de l'autre. Ainsi le mode d'appréhension du monde chez Ducharme se réalise, au niveau de l'écriture, selon le système du syllogisme qu'on peut résumer de la façon suivante:
A. Postulat: "Tout m'avale"
B. Prémisses ou démonstration 
a) majeure: je suis avalée de l'intérieur

«Quand j'ai les yeux fermés, c'est par mon

ventre que je suis avalée..."

b) mineure: je suis avalée par l'extérieur

"Quand j'ai les yeux ouverts, c'est parce que

je vois que je suis avalée..."

C. Conclusion: C.Q.F.D.

"Que j'aie les yeux ouverts ou fermés, je

suis englobée..."

Ainsi résumés, les propos de Bérénice semblent fonctionner selon une logique implacable. Or la lecture complète de ce court texte révèle sinon des failles du moins des excroissances bizarres par rapport à ce raisonnement: la majeure ne fait l'objet d'aucun commentaire; Bérénice "étouffe" un point c'est tout. La mineure, pour sa part, (je suis avalée par l'extérieur) fait l'objet de neuf lignes de commentaires où l'on constate que la technique du syllogisme n'est peut-être, pour Bérénice, qu'un mécanisme de défense. Regardons de plus près.

Les explications supplémentaires de la mineure n'ont, en tant que telles, aucune importance particulière. Elles marquent simplement une différence quantitative par rapport à la majeure. La première phrase ( «Je suis avalée par le fleuve trop grand, par le ciel trop haut, par les fleurs trop fragiles, par les papillons trop craintifs, par le visage trop beau de ma mère ") est dans ce sens pure démonstration ou, si l'on préfère, excédent d'explication. Le narrateur va du général au particulier: fleuve (eau) $\rightarrow$ ciel (air) $\rightarrow$ fleurs (un élément végétal de la terre) $\rightarrow$ papillons (un insecte qui vole dans les airs et se pose sur les fleurs) $\rightarrow$ visage trop beau de ma mère (un élément non seulement qualifié mais aussi particularisé).

Cette phrase est donc en soi tout à fait logique. Celles qui suivent par contre le sont moins. Car dès l'instant où "le visage trop beau de ma mère" est introduit dans le texte, ce dernier se met à disfonctionner. Le syllogisme se transforme subitement en raisonnement tautologique:

(Majeure) Le visage de ma mère est beau pour rien.

(Mineure) S'il était laid, il serait laid pour rien.

(Conclusion) Les visages, beaux ou laids, ne servent à rien.

Le syllogisme, en somme, ne sert à rien parce qu'il est l'objet d'une censure. Le déplacement qu'effectue le narrateur consiste à articuler son syllogisme non pas à partir du substantif et de l'adverbe mais à partir du qualificatif. Ce qui importe, de fait, c'est le “visage en trop» de la mère (le mot "trop " est d'ailleurs le point commun à tous les éléments qui nous sont donnés à lire). Qu'il soit beau, laid ou insignifiant importe peu puisque de toute façon il sera toujours trop (là est l'adverbe censuré) beau, trop laid, trop insignifiant. Le syllogisme tourne donc en rond pour cette raison mais le texte ne peut empêcher le retour du refoulé dès les lignes suivantes:

On regarde un visage, un papillon, une fleur et ça nous travaille, puis ça nous irrite. Si on se laisse faire, ça nous désespère. II ne devrait pas y avoir de visages, de papillons, de fleurs. 
On peut d'abord noter que le matériel utilisé avant le raisonnement tautologique revient en force mais élargi cette fois-ci au niveau de la généralité (un visage, un papillon, une fleur). L'expression-clé «visage trop beau de ma mère "est, elle aussi, transformée en un terme générique et neutre. Cette modification n'a pu être réalisée que parce qu'il y avait eu précédemment l'affirmation «Les visages beaux ou laids ne servent à rien". Pourtant on s'explique mal pour quelle raison «un visage, un papillon, une fleur [...] ça nous travaille, puis ça nous irrite [...] ça nous désespère». S'ils ne servent à rien, s'ils sont indifférents comment pourraient-ils provoquer le désespoir? Pour quel motif valable pourrait-on invoquer, au moyen d'un «il » impersonnel, qu' «il ne devrait pas y avoir de visages, de papillons, de fleurs $»$ ?

La tautologie joue un rôle des plus importants dans le texte. Elle a pour fonction d'annuler l'effet de sens produit par "le visage trop beau de ma mère". Le narrateur fait marche arrière. II essaie d'annuler l'affirmation par un jeu de fausse logique («Les visages beaux ou laids ne servent à rien") mais n'y parvient pas puisque le "trop» revient en force mais masqué par l'ensemble des éléments mis en commun (visage, papillon, fleur) avec le résultat que cette dénégation du "visage trop beau de ma mère" se transforme en une synecdoque où ce n'est plus le visage en trop de la mère qui devrait être anéanti mais tous les visages, tous les papillons, toutes les fleurs.

La dénégation entraîne, par formation réactionnelle, une affirmation généralisée non seulement irrationnelle (pourquoi ne devrait-il pas y avoir de visages, de papillons, de fleurs?) mais extrêmement violente. Le raisonnement de Bérénice est le suivant: puisque le visage de ma mère est trop beau (ou de trop) alors tout m'irrite et me désespère. "Tout m'avale" équivaut à “ ma mère m'avale».

Ce raisonnement spécieux indique que, pour Bérénice, la mère est le tout. Dans cette perspective on pourrait donc être justifié de penser que le fonctionnement psychique de Bérénice est articulé à partir du Moi-Tout (cf. Gérard Mendel, la Révolte contre le père, et Mélanie Klein), c'est-àdire sous le mode régressif (au stade oral, est-il nécessaire de le préciser?) où l'enfant est incapable de prendre une certaine distance par rapport à la mère et dont le processus de fonctionnement s'articule toujours à partir d'une formule binaire type: je t'avale et/ou tu m'avales, je te tue et/ou tu me tues, etc.

La suite du texte montre bien qu'il s'agit de ce type de fonctionnement vécu sous le mode agressif. Bérénice fait tout pour se défaire de l'emprise englobante de sa mère. Incapable de trouver un allié en son père (dans le texte, le mot désespère peut être considéré comme un signifiant-condensation où "père" et "désespoir" sont inexorablement liés), elle tue symboliquement sa mère en la traitant du nom horrible de «chat mort" qui se transforme par la suite en “chamomor» (il faut à tout prix tuer la chatte trop douce mais trop indifférente, la transformer en chameau... même s'il mord!). 
Puisqu'il ne s'agit pas dans ce court article d'étudier tout le roman mais plutôt l'extrait déjà cité, il importe de tirer à son sujet les conclusions qui s'imposent. A ce titre, mon analyse confirme les intuitions du début à savoir que chez Ducharme le mot ou du moins le système qui les lie entre eux prime sur la chose. Dans le texte analysé il est clair que le "visage trop beau de ma mère" et les effets inconscients qu'il pourrait faire naître font l'objet d'une dénégation ratiocinante. Tout se passe comme si le contenu affectif lié au "visage de ma mère" obligeait le narrateur à une sorte de désinvestissement sous la forme d'une production artificielle de concepts ( «les visages, beaux ou laids, ne servent à rien, etc.).

L'expression «parti pris des mots» trouve ici sa justification à propos du travail de l'écriture chez Ducharme. Les mots servent à masquer la chose. Il suffit pour y parvenir de faire des jeux de mots, des calembours, des bons ou des faux raisonnements, etc.

Évidemment ce mécanisme de formation réactionnelle n'empêche pas pour autant le retour du refoulé. II s'agit d'un mécanisme qui a pour fonction de repousser, de déplacer l'intensité de l'affect. II constitue l'élément essentiel du fonctionnement de son écriture. $A$ ce sujet, notons que ce mécanisme occupe une bonne partie du texte. On peut, par extrapolation, présupposer qu'il en est de même pour l'ensemble des écrits de Ducharme.

Le refoulé réapparaît mais de façon déguisèe. Ce nest pius la mere et son visage trop beau qui ne devrait pas exister mais "des visages, des papillons, des fleurs». Ce déplacement du particulier au général possède cette caractéristique d'élargir l'agressivité de Bérénice à l'égard de sa mère sur l'ensemble des choses. Le mécanisme de défense, s'il réussit dans un premier temps (grâce à la phrase neutre «Les visages beaux ou laids ne servent à rien ") à désinvestir l'image abhorrée de la mère, ne l'annule pas pour autant puisqu'il refait surface dans la formule "ll ne devrait pas y avoir de visages...".

Le deuxième paragraphe du texte par son effet de redondance confirme les hypothèses émises précédemment. Qu'on y regarde de plus près.

Le dernier paragraphe se clôt comme suit:

Que j'aie les yeux ouverts ou fermés, je suis englobée: il n'y a plus assez d'air tout à coup, mon ccur se serre, la peur me saisit.

Cette conclusion confirme l'échec du mécanisme de défense que nous venons de décrire. Le résultat est clair: la narratrice aura beau se répéter que «les visages, les papillons, les fleurs ne servent à rien et ne devraient pas exister» cela ne l'empêchera pas pour autant d'étouffer, d'avoir le ccour serré et d'être saisie par la peur.

Devant l'échec de ce faux raisonnement et la finale qui l'accompagne, on devrait normalement s'attendre à ce que le narrateur embraie sur cette notion d'angoisse et de peur. Or c'est exactement le contraire qui se produit. 
La première phrase du texte peut être définie comme le modèle de la phrase insignifiante et, n'était-ce de celle qui suit, on serait en droit de se demander quel talent peut avoir Réjean Ducharme. Car en écrivant: "L'été, les arbres sont habillés. L'hiver, les arbres sont nus comme des vers", Ducharme ne fait rien d'autre que d'écrire des inepties à moins de lire la phrase non pas au niveau de son contenu mais au niveau formel c'est-à-dire en la prenant comme masque et reproduction des phrases types (pour la plupart binaires) sur le bonheur, telle: «Heureux un jour, malheureux le lendemain».

Ici encore il y a déplacement. Au “j'ai peur" succède, au lieu d'une description du fantasme d'angoisse, une série de mots insignifiants qui sont en même temps producteurs de sens. Ducharme annule l'angoisse par la production à vide de mots dont le sens masqué pourrait être: Aujourd'hui j'ai peur, mais demain ce sera fini. Mais en utilisant le formulant "L'été les arbres sont habillés. L'hiver, les arbres sont nus comme des vers", il ouvre toute grande la voie au dérapage.

Du moins est-ce ainsi que le texte fonctionne: une série de phrases sera produite par association dont le résultat sera d'éloigner de plus en plus le narrateur du «la peur me saisit " qui clôturait le premier paragraphe.

Ainsi, «les arbres sont nus comme des vers" engendre "ils disent que les morts mangent les pissenlits par la racine ". Cette phrase n'a aucun sens par rapport à la première à moins d'admettre que "vers" (lui-même né de "hi-ver") ait provoqué chez Ducharme l'association non écrite, "les cadavres sont rongés par les vers $"$, permettant de la sorte à celle qui est donnée de prendre place dans le texte. La même démarche explique l'apparition de la quatrième phrase "Le jardinier a trouvé deux vieux tonneaux dans son grenier ". La racine de pissenlit engendre celle de jardinier dont le rôle est modifié. II n'entretient pas le gazon en arrachant les pissenlits par la racine mais, apparemment sans raison, "a trouvé deux vieux tonneaux dans son grenier ". Ce saut de pissenlit à tonneau est plus obscur mais il pourrait trouver une explication si l'on voulait bien faire comme le jardinier et couper le tonneau en deux (= ton/eau). Pissenlit (pisser au lit) engendre par un jeu subtil de signifiants «ton eau" (ton pipi), tonneaux dans lesquels les ois/eaux viennent boire!

La suite du texte se termine par une anecdote sans intérêt sinon qu'elle s'articule sur les phrases qui précèdent et permet, elle aussi, d'évacuer "la peur me saisit". Le deuxième paragraphe peut donc être considéré comme une production à vide. Il faut attendre le troisième paragraphe pour retrouver l'affect censuré.

Les deux premières phrases de ce paragraphe s'énoncent comme suit: "Je suis seule et j'ai peu [il s'agit d'une coquille de mon édition, la suite du texte indique clairement qu'il faut lire «j'ai peur»]. Quand j'ai faim. je mange des pissenlits par la racine et ça se passe.»

II est clair que le deuxième paragraphe n'a pas annulé la peur. Celleci réapparaît dans le troisième paragraphe, comme c'était d'ailleurs le cas 
pour "le visage trop beau de ma mère" sous la forme d'un principe naturel («Quand j'ai faim, je mange des pissenlits par la racine et ça se passe " et dont l'efficacité est démontrée à partir de plusieurs exemples («Quand j'ai soif, je plonge mon visage dans l'un des seaux et j'aspire. [...] L'hiver, quand j'ai froid, je rentre et je mets mon gros chandail bleu ", etc., etc.) avec le résultat saugrenu que la démonstration du narrateur ne rime à rien puisque cette loi générale ne peut s'appliquer au phénomène de la peur:

On ne peut rien contre la solitude et la peur. Rien ne peut aider. La faim et la soif ont leurs pissenlits et leurs eaux de pluie. La solitude et la peur n'ont rien. Plus on essaie de les calmer, plus elles démènent, plus elles crient, plus elles brûlent.

En somme, il s'agit de la même conclusion que pour le premier paragraphe. La machine à mots fonctionne à merveille mais elle ne mène nulle part. Elle produit du texte. Elle reporte l'angoisse mais ne l'annule jamais.

Alors pourquoi Ducharme propose-t-il cette mécanique ludique. Peutêtre est-ce parce que c'est sa seule porte de sortie. Refusant avec véhémence le réel, il surinvestit les mots tout en sachant pertinemment que les mots ne peuvent rien contre le réel. Tout au plus permettent-ils de donner l'illusion d'une certaine indépendance vis-à-vis des choses. Mais il s'agit bel et bien d'un leurre puisque de toute façon les héros-narrateurs de Ducharme sont toujours prisonniers d'un réel qui les aécœure".

Pourtant Bérénice, de même que tous les héros-narrateurs des romans de Ducharme, privilégie les mots au détriment des choses. Révoltés contre le système ils ont décidé de créer un univers imaginaire, un château de mots dans lequel ils s'enferment à clef se persuadant, sans jamais réellement y parvenir, qu'il ne s'agit pas là d'une pure création de l'esprit.

Croire aux mots au détriment des choses, y croire sans totalement y croire, telle est la contradiction qui soutient la dynamique de l'écriture ducharmienne. A ce titre on peut la définir comme étant du type schizoïde dans la mesure même où cette écriture sans être réellement désarticulée (elle serait alors qualifiée de schizophrénique) propose tout de même une utopique régression au monde de l'enfance et entretient, en accord avec cette régression, l'illusion de la surpuissance des mots dans un climat de violence qui inquiète parfois. Bérénice caresse un rêve de toute-puissance, celui de créer sa propre langue, une langue qui soit une partie d'elle (le bérénicien), une langue qui la place au centre du monde et qui rejette dans les ténèbres le reste de l'humanité. Se pourrait-il que ce rêve de Bérénice de reconstruire le monde à son désir, que ce rêve à la idi Amin Dada ait pu être partagé par Réjean Ducharme?

Nous connaissons heureusement sa réponse: les mots ne servent à rien!

Peut-être... 


\section{Beaulieu: le parti pris des choses}

Si l'écriture de Ducharme déporte constamment la prise en charge du fantasme, celle de Victor-Lévy. Beaulieu l'appelle de toutes ses forces. L'extrait cité parallèlement à celui de Ducharme est éloquent à ce sujet. La logique y est bannie. Abel Beauchemin comprend tout à coup qu'il va mourir. Pourquoi cette pensée lui vient-elle à l'esprit? Nul ne saurait le dire. La certitude l'a frappé au “beau milieu» d'une phrase sans raison apparente sinon qu' «il cherchait ses mots et n'était pas satisfait de ceux qu'il trouvait ". Est-il besoin de dire qu'il y a une distance incommensurable entre le fait de chercher ses mots et la certitude d'une mort prochaine! La réaction d'Abel est brutale et irrationnelle. Pourtant, il s'acharne à y trouver une explication. Les mots, signes abstraits, soudainement s'enflent, se mettent à bouger, acquièrent une densité nouvelle et effrayante. Le texte se déroule alors sous le signe de l'hallucination.

D'un point de vue linguistique, cette hallucination est une métaphore filée dont la principale caractéristique est de rendre éminemment concret ce qui à l'origine était abstrait. Les mots tracés par Abel se métamorphosent d'abord en serpents, ensuite en un tronc de vieil érable puis finalement en d'inquiétantes étoiles noires.

Si la première métaphore, celle où les mots trop longs deviennent des «serpents à lettres pâteuses qui entouraient ses jambes et faisaient monter le sang à sa tête" justifie minimalement le sentiment de mort éprouvé par le narrateur, la seconde ne fait qu'évoquer l'image de la mort dans celle de l'arbre.

Quant à la troisième métaphore, elle est, elle aussi, peu explicative au sujet du sentiment qu'éprouve le narrateur. Que les mots brillent trop devant ses yeux et que de ce fait ils se transforment "en d'apeurantes étoiles noires qui glissent silencieusement derrière ses paupières closes", cela prouve simplement qu'Abel est sujet à des hallucinations, qu'il risque la psychose mais non pas qu'il va mourir.

Pourtant Abel après cette série de fantasmes "se laiss[e] tomber dans sa chaise et se me[t] à hoqueter ". Pour lui tout est fini!

La réaction est si absurde que dans la deuxième partie du paragraphe, le narrateur amorce une nouvelle explication que, de façon tout à fait incompréhensible, il laisse brutalement en plan.

La phrase débute par "quelque chose en lui..." puis est coupée par une longue parenthèse de dix lignes qui la clôt. Le résultat est étonnant: le "quelque chose en lui " n'a ni verbe ni complément. II reste en l'air! Amputé! En sorte qu'on peut affirmer que ce "quelque chose en lui » est frappé d'une censure d'autant plus évidente que la phrase n'est grammaticalement pas française.

Il est clair que «quelque chose en lui» est annulé par la parenthèse. Cela est visible autant au niveau formel qu'au niveau de la signification. "l est peut-être utile de reproduire la uphrase". 
[...]; quelque chose en lui - (mais peut-être aussi cela venait-il de l'extérieur, dans cette fourmi absurde se frappant obstinément la tête contre la vitre de la fenêtre, dans une entreprise désespérée tout autant qu'insensée et dehors les sous-vêtements bruns battaient au vent, faisant quelque danse obscène sur la corde à linge, provoquant l'œil, créant dans la cour un champ d'angoisse parfaitement circulaire et au centre duquel, comme quelque bete suant et jurant, lui, Abel Beauchemin venait de comprendre que jamais plus il ne pourrait écrire de romans).

Du reste, si cette phrase incomplète ne frappe pas immédiatement le lecteur cela tient au fait qu'elle est structurée de telle sorte que l'on en oublie le premier membre magnifiquement bien récupéré par les deux dernières propositions de la parenthèse. Ainsi la phrase débute et se termine par le mot lien "lui»: "quelque chose en lui $-[\ldots]$ lui, Abel Beauchemin venait de comprendre que jamais plus il ne pourrait écrire de romans".

Grâce à cette entorse aux lois grammaticales, l'angoisse de mort ne vient plus de lui mais bien de l'extérieur. Le "quelque chose en lui" subit une dénégation violente malgré la tournure interrogative de la proposition qui suit ( «mais peut-être aussi cela venait-il de l'extérieur...»). La phrase ainsi structurée équivaut plutôt à une formulation type «ce n'est pas moi, ce sont les autres». C'est d'ailleurs en ce sens que l'on peut définir l'écriture de Victor-Lévy Beaulieu comme étant de type paranoïde. Ainsi malgré une affirmation qui, au début, semble insinuer le contraire ("quelque chose en lui...") l'angoisse de mort vient, du moins aux yeux du narrateur, de l'extérieur, de l'autre. Ce mécanisme, est-il nécessaire de le dire, est fondamental dans la paranoïa.

L'angoisse du narrateur, au lieu de connaître une explication intrapsychique, se projette de fait sur les choses immédiatement perceptibles pour lui. Ce sont d'abord les lettres qu'il trace au moment même où l'angoisse le saisit. Elles deviennent tour à tour «serpents», «tronc d'arbre " et "étoiles apeurantes" c'est-à-dire créatrices de fantasmes dont au moins un renvoie à l'enfance du narrateur. Puis, il faut croire que le narrateur a levé la tête, c'est la fourmi à la fenêtre qui se trouve investie et finalement c'est la cour extérieure (dehors les sous-vêtements bruns battaient au vent, faisant quelque danse obscène $"$ ).

La description de l'angoisse du narrateur procède donc selon un mouvement excentrique. Elle, qui était pourtant née de lui, est extirpée de sa personne et investie dans les choses qui l'entourent. Ce jeu de désinvestissement-investissement produit, dans le cas qui nous intéresse, un résultat pour le moins étrange: le narrateur, à la fin du texte, n'est plus là où il est mais au centre de la cour (lui qui avait été saisi d'angoisse au beau milieu d'une phrase!): "créant dans la cour un champ d'angoisse parfaitement circulaire et au centre duquel, comme quelque bête suant et jurant, lui, Abel Beauchemin venait de comprendre que jamais plus il ne pourrait écrire de romans ".

Le narrateur bénéficierait-il du pouvoir d'ubiquité? On peut se poser la question. Chose certaine, il se complaît dans la projection. À cause 
d'elle nous ne saurons rien de son angoisse réelle. Derrière le masque de l'aveu (V.-L. Beaulieu donne toujours l'impression de faire une éternelle confession scabreuse) se cache la vraie réponse. Ce «quelque chose en lui " sans doute ne le connaîtrons-nous jamais puisqu'Abel interpose des choses entre lui et nous qui empêchent le véritable aveu. Ce sont elles les vraies responsables de son angoisse.

Voilà pourquoi il ne faut pas se laisser prendre au piège qu'il nous tend. Si on voulait lire le texte d'Abel Beauchemin selon la méthode psychanalytique il faudrait se garder de prendre pour acquis l'apparente absence de censure du narrateur. Car à y regarder de près on constate bien que son écriture est piégée. A preuve "ce quelque chose en moi " grossièrement censuré.

II suffirait de relire le texte que je viens d'analyser avec cette attention flottante si chère à Freud pour constater que dans ce court paragraphe la répétition obsédante s'y installe en toute sécurité. Entre la première moitié du paragraphe et la seconde la même image s'impose d'emblée: celle du «père jurant et crachant le brun-malade de sa chique" qui réapparaît morcelée et pleine d'ambiguïté alors que «les sous-vêtements bruns battaient au vent, faisant quelque danse obscène sur la corde à linge" et que lui pauvre Abel, "comme quelque bête suant et crachant", "venait de comprendre que jamais plus il ne pourrait écrire de romans ".

Pourquoi, alors que son père flotte probablement sur la corde à linge, pourquoi en suant et crachant comme lui, Abel devrait-il connaître la stérilité et même la mort?

Bessette, dans Trois romanciers québécois a donné une certaine réponse à cette question. II y aurait beaucoup à dire encore sur cette image du père qui plane (ou flotte!) sur la conscience d'Abel comme sur celle de beaucoup de héros des romans de V.-L. Beaulieu. Car si Ducharme est obsédé par la mère, Beaulieu est surtout préoccupé du père. Cela détermine deux types d'esthétique: celle de Ducharme privilégie le vide, le trou; celle de Beaulieu, le plein, la chose (celle, énorme, que Jos Connaissant tient entre ses mains ou encore ce que ce même Jos appelle "le mystère du bout de la queue de Christ ").

II s'agit évidemment d'un jugement à l'emporte-pièce que, malgré tout, notre analyse confirme. À ce titre la remarque est intéressante. Elle nous renseigne sur une certaine asexualisation du texte de Ducharme et sur une sursexualisation du texte de Beaulieu. La "chose» chez Beaulieu se multiplie: les pénis prennent beaucoup de place dans son œuvre (cf. ceux des hommes, des étalons, des chiens, etc., etc.). Cette fascination du phallus explique aussi le thème de l'homosexualité que Gérard Bessette a développé en lui donnant une interprétation qui me paraît suspecte. Car si on veut bien admettre mon affirmation selon laquelle l'écriture de V.-L. Beaulieu est de type paranoïde, force nous est d'admettre que l'homosexualité tout comme l'image omniprésente du père constituent des thèmes privilégiés pour ce type de fonctionnement psychique (le cas Shreber étudié 
par Freud et celui de Strinberg ${ }^{8}$ analysé par Janine Chasseguet-Smirgel sont éloquents à ce sujet). Encore faut-il comprendre que dans ces deux cas d'étude de la paranoïa, l'homosexualité constitue la clef de l'interprétation parce qu'il s'agit d'un fantasme violemment refoulé lequel ne peut réapparaître que sous de multiples déguisements. Chez Beaulieu l'écart est encore plus grand: les scènes hétérosexuelles sont beaucoup plus nombreuses que les scènes homosexuelles. En outre les premières sont plus réalistes que les secondes à ce point qu'on peut penser que le fantasme d'homosexualité est en quelque sorte un rêve interdit (la description sur la planète Pluton donnée dans Malcomm Hudd est significative à ce sujet: il s'agit d'un rêve fort ambigu; on peut dire la même chose du voyage en auto vers Montréal raconté pour la Xième fois à Chien Chien Pichlotte par Milien dans les Grands-Pères).

Étant donné que mon analyse ne porte que sur un court texte de Beaulieu il me paraît inapproprié de développer plus longuement cette problématique. Malgré tout, deux éléments dans le texte me semblent à retenir dans le sillage de ce qui précède. D'abord le nom du narrateur qui réunit, non sans une certaine ambiguïté, le masculin et le féminin du mot "beau " (A-bel Beau-chemin) ce qui n'est pas sans intérêt pour la problématique de l'homosexualité; d'autre part la récurrence de cet étrange mot "brun" (le «brun-malade de sa chique" et les «sous-vêtements bruns") qui laisse supposer, ce qui est tout à fait normal dans le cas d'une écriture paranoïde, le surinvestissement de la fonction anale (alors que chez Ducharme c'était la fonction orale qui s'imposait).

\section{Prospective}

Comme on a pu le constater, les deux courts extraits retenus livrent un matériel énorme par rapport à mes hypothèses de recherche. Il faudrait bien sûr élargir l'analyse et vérifier sa pertinence sur l'ensemble des cuvres des deux auteurs de même que sur celles d'autres auteurs québécois. L'analyse qui a été faite permet de croire que celles qui sont à venir pourraient être riches de découvertes.

André Vanasse

UQAM

1. Ferdinand de Saussure, Cours de linguistique générale, Paris, Payot, 1969, p. 98.

2. Gilles Marcotte, "Réjean Ducharme contre Blasey Blasey", in Études françaises, vol. 11, nos 3-4, p. 247-284.

3. Bernard Dupriez, "Ducharme et des ficelles", in Voix et images du pays V, Montréal, Les Presses de l'Université du Québec, 1972, p. 165-185.

4. Gérard Bessette, "Victor-Lévy Beaulieu", in Trois romanciers québécois, Montréal, Éditions du Jour, 1975. 
5. André Vanasse, «A la recherche du mystère dy bout de la queue de Christ", in Livres et auteurs québécois 1972. Montréal, Editions Jumonville. p. 385-396.

6. Gabrielle Poulin, dans la revue Relations du mois de septembre 1972 ("De la castration à l'incontinence: les premiers romans de Victor-Lévy Beaulieu", p. 249-251) et celle du mois de novembre de la même année («Folie ou mysticisme? - Victor-Lévy Beaulieu w. p. 312-314).

7. S. Freud, «Remarques psychanalytiques sur l'autobiographie d'un cas de paranoïa: Dementia Paranoides (Le président Schreber), in Cinq psychanalyses, Paris, PUF, 1970, p. 263-324.

8. Janine Chasseguet-Smirgel, “A propos d'Auguste Strindberg: contribution à l'étude de la paranoïa", in Pour une psychanalyse de l'art et de la créativité, Paris, PBP, 1971, p. 107-164. 